\title{
Investigation of Luminescence Processes in YAG Single Crystals Irradiated by 50 MEV Electron Beam
}

\author{
V.V. Harutyunyan ${ }^{1}$, E.M. Aleksanyan ${ }_{2}^{1}$ V.C. Baghdasaryan ${ }^{1}$, P.B. \\ Kostanyan $^{2}$, G. Bondarenko ${ }^{3}$, M. Kirm ${ }^{4}$, S. Vielhauer ${ }^{4}$ \\ ${ }^{I}$ A. Alikhanian National Scientific Laboratory, Yerevan, 0036, Armenia \\ ${ }^{2}$ Institute for Physical Research, Armenian National Academy of Sciences, 0203 Ashtarak-2, Armenia \\ ${ }^{3}$ National Research University "High School of Economics", (NRU HSE), Moscow, Russia \\ ${ }^{4}$ Institute of Physics, University of Tartu, Riia 142, 51014 Tartu, Estonia \\ *E-mail: vachagan.harutyunyan@aanl.am
}

Received 8 October 2019

\begin{abstract}
Absorption, emission and excitation spectra of $50 \mathrm{MeV}$ electron beam irradiated and asgrown $Y A G$ single crystals were studied and compared in the 10-300 $\mathrm{K}$ temperature range using timeresolved luminescence spectroscopy under UV/VUV/XUV excitation by synchrotron radiation and cathodoluminescence. The emission spectra consist of intrinsic (excitonic) and defect related nonelementary bands in the VIS/UV range. It is shown that fast electrons create stable $F$ and $F^{+}$color centers with characteristic emission and absorption bands in the visible/UV range. Induced absorption caused from these defects starts at $4.2 \mathrm{eV}$. Energy transfer from host to color centers is not efficient process.
\end{abstract}

Keywords: YAG, electron-irradiation, F and F+ centers, luminescence

\section{Introduction}

Single crystals of yttrium aluminum garnet $Y_{3} A_{5} O_{12}(Y A G)$ are well studied due to their excellent mechanical, optical and chemical properties. $Y A G$ crystals and ceramics are widely used as hosts for doping with different rare-earth ions for use in different applications like laser crystals, fast scintillators, phosphors in LEDs, dosimeters for ionizing radiation etc. (e.g. Haranath et al., 2006, Lu et al., 2002). The study of luminescence properties of pure and rare earth (RE) doped YAG crystals in our previous works (Aleksanyan et al., 2009, Ning et al., 2007) has revealed that the efficiency of the host - RE-ion energy transfer plays important role in these matrices. Although this material is widely used under various radiation conditions, only limited data on its related properties are available in literature (Jiang, 2006). In particular the role of defects in the relaxation processes of electronic excitations in electron irradiated $Y A G$ crystals is not understood in detail.

In binary and complex metal oxides, electron irradiation causes formation of stable defects, where the simple $F$ and $F^{+}$centers are most commonly observed, but also more complex entities can be formed during growth, irradiation or thermochemical treatment.

Radiation-induced defects in $Y A G$ had been under investigation earlier by a number of authors (e.g. Pujats et al., 2001, Popov et al., 2010 and references therein, Zorenko et al. 2011). 
Different studies suggest that luminescence bands of $F$ and $F^{+}$centers peak at 2.7 and $3.1 \mathrm{eV}$ (Pujats et al., 2001) with related absorption bands at 5.16,6.3, and 3.35,5.27 eV, respectively. Ashurov et al. (2001) proposed that under neutron irradiation $F^{+}$centers are created with typical emission at $2.38 \mathrm{eV}$. There are data available from various sources listed above not being in a complete agreement.

With our study of as-grown and $50 \mathrm{MeV}$ electron irradiated $Y A G$ crystals we would like to bring additional clarification to the role of defect states and relaxation processes of electronic excitations created by ionizing radiation in the UV-XUV range and by charged particles.

\section{Experimental details}

Nominally pure $Y A G$ crystals were grown by the Czochralski method. Their irradiation with $50 \mathrm{MeV}$ electrons took place at $263 \mathrm{~K}$ using an accelerator at Yerevan Physics Institute. During irradiation the sample was cooled with gaseous nitrogen to avoid overheating and possible mechanical damage. The samples were exposed to an average irradiation dose of $10^{17} \mathrm{el} / \mathrm{cm}^{2}$. Two polished $Y A G$ plates of identical size and thickness were studied at the experiments described below.

Low temperature time-resolved VUV spectroscopy with synchrotron radiation was performed at the SUPERLUMI station of HASYLAB at DESY, Hamburg (Zimmerer, 2007). Emission spectra in the spectral range 200-700nm were recorded under excitation by $330-50 \mathrm{~nm}(3.75-25 \mathrm{eV})$ photons. XUV measurements were carried out using a mobile setup at the BW3 beam-line described in (Kirm, 2003) using a $0.27 m$ SPEX $270 \mathrm{M}$ spectrometer with $25 \mathrm{~nm} / \mathrm{mm}$ dispersion and liquid $N_{2}$ cooled Princeton Instruments CCD. A fiber optics was used in luminescence collection. The luminescence spectra are not corrected for the sensitivity of detection channel.

Optical absorption spectroscopy was studied at RT in a spectral region of $6.5-1.5 \mathrm{eV}(190-850 \mathrm{~nm})$ using a double-beam spectrophotometer JASCO V-540 in the Institute of Physics in Tartu. Cathodoluminescence spectra in the temperature range of $5-300 \mathrm{~K}$ were recorded using a home-built setup with variable energy of the electron-beam (5-30keV) in Tartu (Feldbach, et al. 2006), where luminescence can be analyzed in VUV and UV-visible range using two different monochromators. The recorded spectra are corrected to the transmission of analyzing channel and detector sensitivity.

\section{Results and discussion}

The absorption spectra of pure $Y A G$ show a steeply rising intrinsic absorption edge near $6.7 \mathrm{eV}$ (Tomiki et al. 1996, Kirm et al. 2000) (see Fig. 5). The energy gap of YAG has been determined to be $8.0 \mathrm{eV}$ (Kirm et al. 2000 and references therein). The comparison of absorption 
spectra of nominally pure and electron-irradiated $Y A G$ crystals at RT shows that induced absorption by electron irradiation starts at $\sim 4.5 \mathrm{eV}$ (Fig. 1), where the contribution of the color centers is expected in the transparency range of $Y A G$. It is in agreement with absorption induced in neutron-irradiated $Y A G$ at RT (Ashurov et al., 2001). However, the absorption bands of $F$ and $F^{+}$ centers cannot be distinguished either in absorption (Fig. 1) or in excitation (Fig. 5).

Fig. 2 shows luminescence emission spectra of irradiated $Y A G$ crystal at different temperatures, excited with XUV photons. Spectra consists of two main bands in UV $(3.6-4.8 \mathrm{eV})$ and visible range $(2.0-2.4 \mathrm{eV})$ at different temperatures. Similar results have been obtained also with cathodoluminescence measurements and will be discussed later. The short wavelength side UV band observable at $10 \mathrm{~K}$ is due to self-trapped excitons (STEs) (Kirm et al. 2000), whereas remaining room temperature emission peaked at $4.0 \mathrm{eV}$ is assigned to the radiative decay of excitons perturbed by antisite defects $\left(Y^{3+}\right.$ ion at $A l^{3+}$ site) (Babin et al, 2005). The visible bands are originating from luminescence of defects. Fig. 2 evidences that there is significant red-shift of both high- and low-energy luminescence bands with increasing the temperature. The shift is visible even at such small temperature difference as $8 \mathrm{~K}$. A possible interpretation is a redistribution intensity of subbands due to the intrinsic STE in favor of several perturbed exciton states near antisite defects as revealed by (Babin et al. 2005). The role of as-grown defects is well demonstrated in Fig. 3, where for non-irradiated $Y A G$ samples $F$-centers emission at $2.7 e \mathrm{~V}$ is dominating under excitation in the related absorption band at $6.2 \mathrm{eV}$. The excitation in host absorption by $6.9 \mathrm{eV}$ photons results in luminescence of perturbed STE without energy transfer to defect states as shown in Fig. 3. According to Smith et al., (2003) the displacement of lattice ions in oxides can take place if the incident electron energy is $0.33-0.35 \mathrm{MeV}$ causing formation of lattice defects by impact. In Fig.3, there is no significant difference between the irradiated and nonirradiated $Y A G$ samples, demonstrating that the creation of additional defect states has no influence on the luminescence under $6.9 \mathrm{eV}$ excitation, which causes creation of intrinsic host excitations.

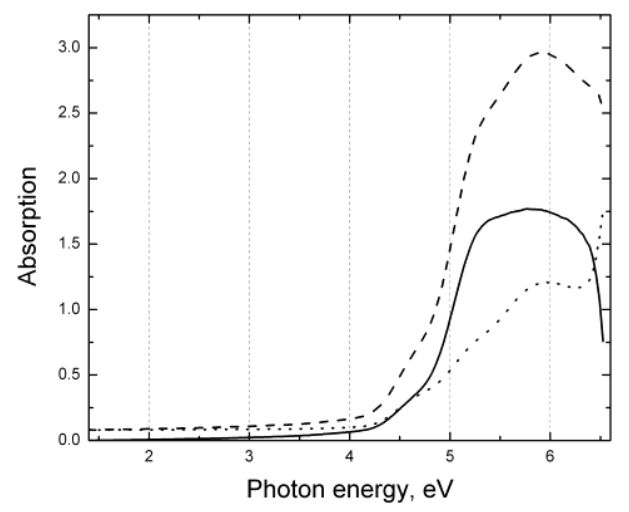

Fig. 1. Absorption spectra of irradiated YAG (dashed line, fluency is $10^{17} \mathrm{e} / \mathrm{cm}^{2}$ ) and non-irradiated YAG plates (dotted line). The induced absorption spectrum (solid line) is obtained by subtracting the latter spectrum from the former one. $\mathrm{T}$ $=300 \mathrm{~K}$. 


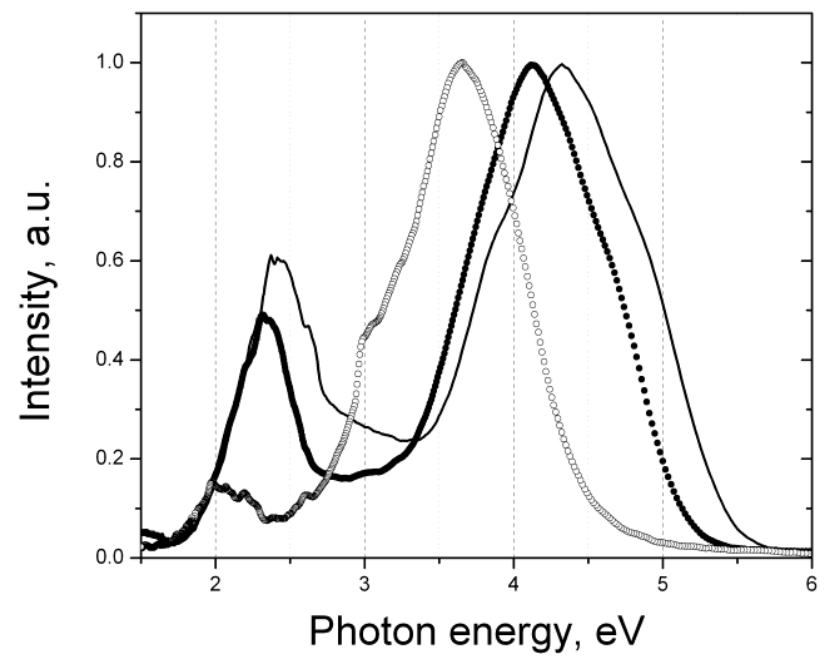

Fig. 2. Normalized emission spectra of $50 \mathrm{MeV}$ irradiated YAG crystal under excitation by $130 \mathrm{eV} \mathrm{XUV}$ photons at room (empty circles) and low (filled circles $=16 \mathrm{~K}$, solid line $=8 \mathrm{~K}$ ) temperatures.

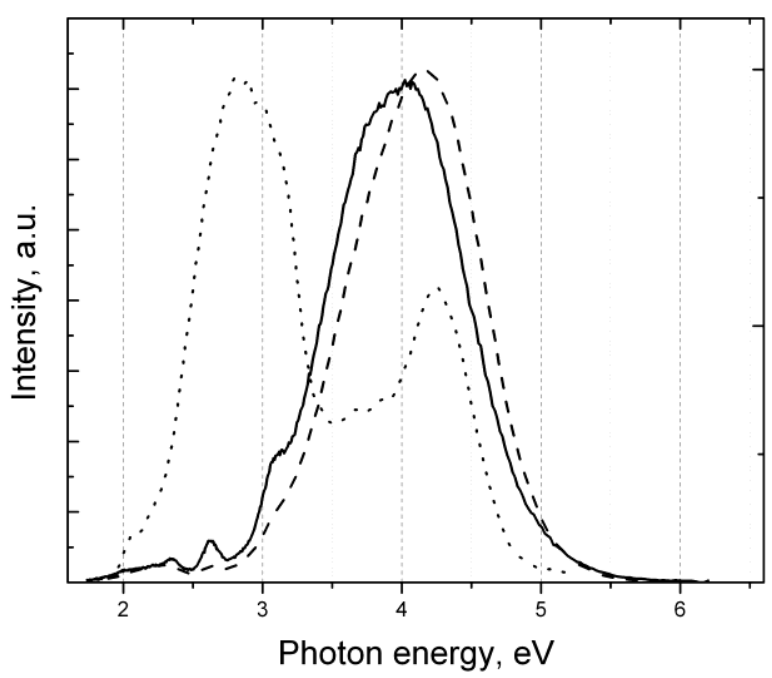

Fig. 3. Normalized emission spectra of non-irradiated (dashed line) and $50 \mathrm{MeV}$ electron irradiated (solid line) YAG crystals at $300 \mathrm{~K}$ excited by $6.9 \mathrm{eV}$ photons and in non-irradiated crystal (dotted line) excited by $6.2 \mathrm{eV}$ photons at 10 $\mathrm{K}$. 


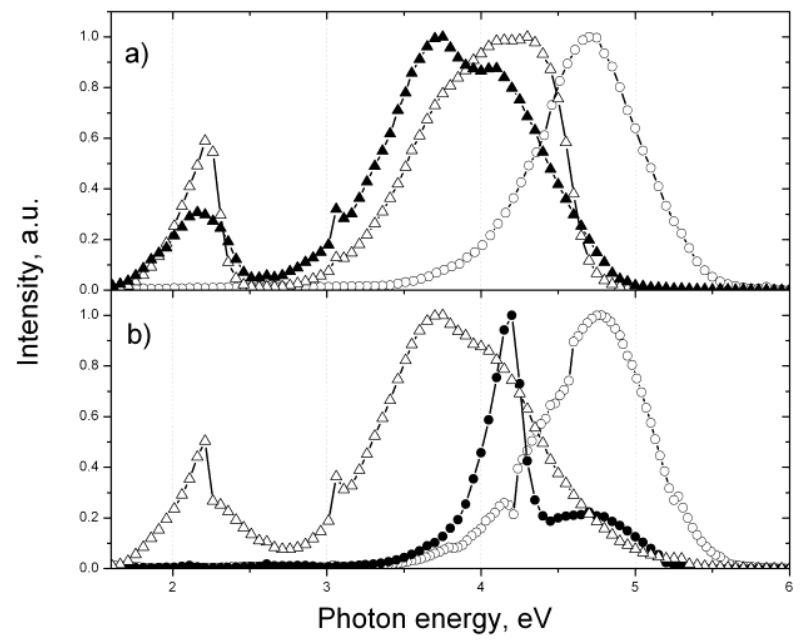

Fig. 4. Cathodoluminescence emission spectra of non-irradiated (a) and $50 \mathrm{MeV}$ electron irradiated (b) YAG crystals. Measurements were carried out at $10 \mathrm{~K}$ (circles) and room temperatures (triangles) before (empty symbols) and after irradiation by $10 \mathrm{keV}$ electron during $100 \mathrm{~min}$ (filled symbols).

Cathodoluminescence (CL) measurements performed at low and room temperatures revealed generally similar structure of broad bands both in non- and electron irradiated samples as under XUV excitation. At low temperatures, luminescence of STE prevails with the maximum near $4.8 \mathrm{eV}$ in both samples. In $50 \mathrm{MeV}$ electrons irradiated crystal there is also one small shoulder at $4.2 \mathrm{eV}$. However, after a few minutes of irradiation by $10 \mathrm{keV}$ electrons during cathodoluminescence measurements, this feature evolves into a luminescence band which becomes dominant and remains stable. This energy of electrons is too small to induce any defects by the impact mechanism. In radiation sensitive alkali halide crystals, another defect creation mechanism, which causes formation of $F$ - and $H$-centers during the decay of self-trapped excitons, plays an important role under VUV excitation (Lushchik et al., 1995). In more radiation resistant oxides, the decay of electronic excitations with stable defect formation is a less common process, although selftrapping occurs as in the case of $Y A G$. Therefore, recharging of previously created defect states (e.g. $F \rightarrow F^{+}+$electron) modifies the optical and dielectric properties as described for alumina by (Vallayer et al., 2001). The induced absorption onset in $Y A G$ is at $4.2 \mathrm{eV}$ (Fig. 1), which means that the luminescence intensity at higher energies is reduced due to reabsorption in electron irradiated sample. As can be seen in Fig. 4 the luminescence band originating from radiative decay of STE is present at lower intensity. In the cathodoluminescence of non-irradiated YAG crystal at low temperatures there is no such band present at $4.2 \mathrm{eV}$, but under excitation in the transparency range (Fig. 3, dotted line) it is well pronounced along with $F$-center emission peaked at $2.8 \mathrm{eV}$. The emission bands near $4.2 \mathrm{eV}$ have been assigned to the radiative decay of exciton states 
localized near antisite defects $\left(Y_{A l}\right)$ at room temperature (Zorenko et al. 2012 and references therein). This also explains its presence in the luminescence spectra of as-grown, non-irradiated $Y A G$ crystals.

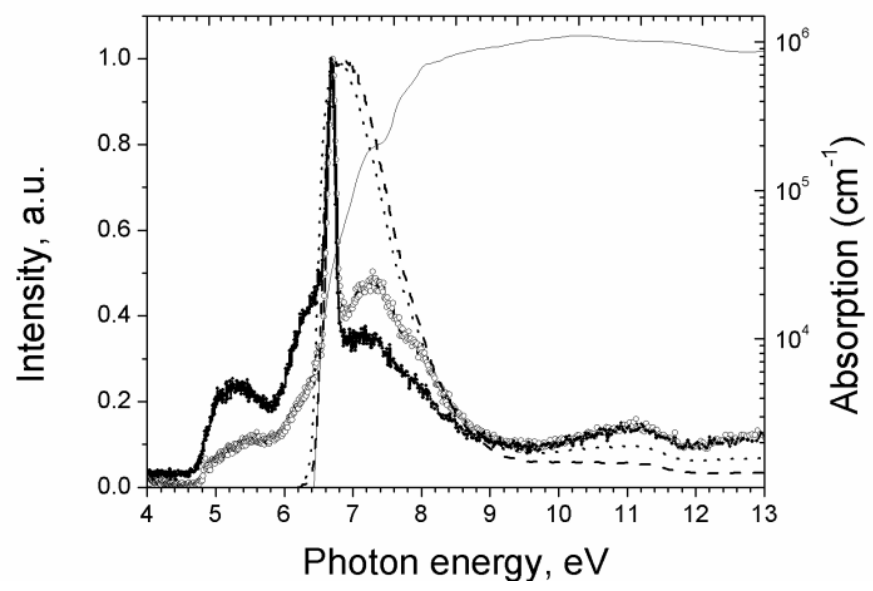

Fig. 5. Excitation spectra of emissions recorded at 2.53 and $3.06 \mathrm{eV}$ from $50 \mathrm{MeV}$ electron irradiated YAG crystal at 8 $\mathrm{K}$ due to the $\mathrm{F}$ (filled circles) and $\mathrm{F}^{+}$(open circles) color centers, respectively. For comparison, the excitation spectrum of excitonic emission is also shown (dashed line) recorded at $4.77 \mathrm{eV}$ from as-grown crystal at $10 \mathrm{~K}$. Solid line represents the absorption spectrum of a pure YAG crystal at $300 \mathrm{~K}$ (Tomiki et al. 1996).

At room temperatures CL emission spectra behave analogously in both samples. In agreement with earlier studies (Babin et al. 2005), the luminescence maxima shift towards lower energies with increasing temperature. It consists of two overlapping bands in the $\mathrm{UV}$ range at 4.2 and $3.7 \mathrm{eV}$ and luminescence in the visible range with maxima near $2.2 \mathrm{eV}$ (Fig.4). The prolonged irradiation of asgrown $Y A G$ at room temperature by $10 \mathrm{keV}$ electrons also results in redistribution of intensities towards the lower energies. In this region the radiative decay of excitons and recombination luminescence near antisite defects is expected (Babin et al. 2005 and Zorenko et al. 2012). The low energy luminescence is assigned to more complex defects, whose nature needs further investigation.

Excitation spectra recorded for the emissions $F$ and $F^{+}$centers show rather similar structure, indicating that the separation of specific absorption of these centers is not possible in $Y A G$ (Fig. 5). The excitation for both types of centers starts at $4.7 \mathrm{eV}$ well below the intrinsic absorption. Both excitation spectra show a minimum at $5.8 \mathrm{eV}$, followed by a strong peak at $6.7 \mathrm{eV}$ near the edge of fundamental absorption. This is due to interplay of penetration depth effects in the regime of low host absorption with exciton formation processes. The region of excitonic absorption extends until $8 \mathrm{eV}$ where color center luminescence is most efficiently excited through energy transfer from exciton states. The intensity of both emissions strongly decreases above $8 \mathrm{eV}$, where electron-pair creation starts. It is confirmed by luminescence spectra (Figs. 2, 3 4) of the present work that electron-hole pair formation resulting from ionizing radiation or particles leads mostly to 
the excitation of self-trapped exciton luminescence either unperturbed or localized near antisite defects. The multiplication processes of electronic excitations (see e.g. Lushchik et al., 1995), where one photon is able to create more than one excitation, can take place at energies higher than $E_{g}+$ defect absorption band $\sim 8+5=13 \mathrm{eV}$. However, the excitation spectra do not show any additional pronounced bands testifying that this process is not active in the studied energy range. The small modulations in the spectra are due to changes in the absorption coefficient, which has as high values as $10^{6} \mathrm{~cm}^{-1}$ in this energy region (Tomiki et al. 1996)

Another interesting observation is that the relative intensity of $F$ and $F^{+}$emissions changes in the transparency and host absorption region and become equal at energies exceeding the band gap. There can be several reasons for such behavior. The ratio of $F$ and $F^{+}$centers has been changed using UV irradiation to ionize $F$ centers in sapphire (Vallayer et al., 2001). In our case we should look more closely to energy transfer processes by excitons. Self-trapping is very fast process prohibiting exciton diffusion, which is one of the main energy transfer mechanisms in systems with free excitons like $M g O$ (Kirm et al. 2003). Therefore the overlap of STE emission with absorption bands of color centers plays significant role. As the $F^{+}$luminescence excitation efficiency increases in the excitonic region, it indicates that situation is more favorable for $F^{+}$centers, with known absorption bands at 3.35 and $5.27 \mathrm{eV}$ according to Ashurov et al. (2001).

Time-resolved luminescence has been very useful in identifying and separating processes due to the $F^{+}$centers with fast ns decay and slower processes in microsecond scale due to $F$ centers. Typical life-times of $F^{+}$centres in $Y A G$ is $2.3 n s$ under intra-centre excitation (Zorenko et al., 2011) and in other metal oxides like $\mathrm{BeO}$ and $\mathrm{Al}_{2} \mathrm{O}_{3}$ are $\tau \leq 1 n s$ and $\tau \sim 2.2 n s$, respectively (Pustovarov et al. 2001). However, in the case of $Y A G$ under host excitation it was not possible to separate $F^{+}$bands, which means that energy transfer from host to color centers is inefficient. The another probable reason lies in the more complex emission spectra due to various centers of intrinsic and extrinsic nature in $Y A G$. Overlapping emissions with different decay times mask each other, interfering with attempts to use pulsed synchrotron radiation to reveal the fast emissions.

\section{Conclusion}

Irradiation by $50 \mathrm{MeV}$ electrons of $Y A G$ crystals results in formation of stable color centers. Induced absorption starts in the UV range where $F$ and $F^{+}$centers are known to absorb. However, highly sensitive luminescence methods were not sufficient to distinguish any color center bands in details. The energy transfer processes from host absorption the color centers are rather inefficient as testified by studies using synchrotron radiation VUV- XUV range and low energy electron-beam excitation. 


\section{Acknowledgements}

Financial support of the Estonian Science Foundation, for Postdoctoral researcher grant no. MJD166 under "Mobilitas" program and research grant no. 8306 are gratefully acknowledged. Help of Drs. A. Kotlov and E. Vasil'chenko for assistance in conducting several experiments is also gratefully acknowledged.

\section{References}

[1] Haranath, D., Chander, H., Sharma, P., Singh, S., 2006. Enhanced luminescence of Y3A15O12:Ce3+ nanophosphor for white light-emitting diodes.

[2] Lu, J., Ueda, K., Yagi, H., Yanagitani, T., Akiyama, Y., Kaminskii, A.A., 2002. Neodymium doped yttrium aluminum garnet (Y3Al5O12) nanocrystalline ceramics - a new generation of solid state laser and optical materials. J. of Alloys and Compounds 341, 220-225.

[3] Aleksanyan, E., Harutunyan, V., Kostanyan, R., Feldbach, E., Kirm, M., Liblik, P., Makhov, V.N., Vielhauer, S., 2009. 5d-4f luminescence of Er3+ in YAG:Er3+. Optical Materials 31, 1038-1041.

[4] Ning, L., Tanner, P.A., Harutunyan, V.V., Aleksanyan, E., Makhov, V.N., Kirm, M., 2007. Luminescence and excitation spectra of YAG:Nd3+ excited by synchrotron radiation. Journal of Lumin. 127, 397-403.

[5] Jiang, Ch., 2006. Neutron irradiation of Czochralski and temperature gradient technique grown YAG crystals. Physica B 373, 42-45.

[6] Pujats, A., Springis, M., 2001. The F-type centers in YAG crystals. Radiation Effects \& Defects in Solids 155, 6569.

[7] Popov, A.I., Kotomin, E.A., Maier, J., 2010. Basic properties of the F-type centers in halides, oxides and perovskites. Nucl. Instr. Meth. B 268, 3084-3089.

[8] Zorenko, Y., Zorenko, T., Voznyak, T., 2011. Luminescence centers in Y3Al5O12:La single crystals, J. Physics: Conf. Ser. 289, 012028.

[9] Ashurov, M.Kh.,Rakov, A.F., Erzin, R.A., 2001. Luminescence of defect centers in yttrium-aluminum garnet crystals. Solid State Communications 120, 491-494.

[10]Zimmerer, G., 2007. SUPERLUMI: a unique setup for luminescence spectroscopy with synchrotron radiation. Radiat. Meas. 42, 859-864.

[11] Kirm, M., Lushchik, A., Lushchik, C., Vielhauer, S., Zimmerer, G., 2003. Luminescence of pure and doped Al2O3 and $\mathrm{MgO}$ single crystals under inner-shell excitation, J. Lumin. 102, 307-312.

[12] Feldbach, E., Kotlov, A., Kudryavtseva, I., Liblik, P., Lushchik, A., Maaroos, A., Martinson, I., Nagirnyi, V., Vasil'chenko, E., 2006. Low-temperature irradiation effects in lithium orthosilicates, Nucl. Instrum. Methods Phys. Res. Sect. B-Beam Interact. Mater. Atoms. 250, 159-163.

[13] Kirm, M., Lushchik, A., Lushchik, Ch., Zimmerer, G., 2000. Investigation of Luminescence Properties of Pure and Ce3+ Doped Y3A15O12 Crystals Using VUV Radiation. in "Physics and Chemistry of Luminescent Materials" C. Ronda, L.E. Shea, A.M. Srivastava Editors, PV 99-40, The Electrochemical Society Proceedings Series, Pennington, NJ, 113-122.

[14] Babin, V., Blazek, K., Krasnikov, A., Nejezchleb, K., Nikl, M., Savikhina, T., Zazubovich, S., 2005. Luminescence of undoped LuAG and YAG crystals, Phys. Stat. Sol. (c) 2, No. 1, 97-100.

[15] Smith, K.L., Colella, M., Cooper, R., Vance, E.R., 2003. Measured displacement energies of oxygen ions in titanates and zirconates, Journal of Nuclear Materials 321, 19-28.

[16] Lushchik, A., Kudryavtseva, I., Lushchik, Ch., Vasil'chenko, E., Kirm M., Martinson, I., 1995. Creation of Stable Frenkel Defects by VUV Radiation in KBr Crystals Under Conditions of Multiplication of Electronic Excitations, Phys. Rev. B 52, 10069-10074. 
[17] Vallayer, J., Jardin, C., Treheux, D., 2001. Optical and dielectric behaviors of alumina after an electromagnetic irradiation, Optical Materials 16, 329-333.

[18]Zorenko, Y., Zorenko, T., Gorbenko, V.V., Voznyak, T., Savchyn, V., Bilski, P., Twardak, A., 2012. Peculiarities of luminescent and scintillation properties of YAG:Ce phosphor prepared in different crystalline forms, Optical Materials 34, 1314-1319.

[19] Tomiki, T., Isa, Y., Kadekawa, Y., Ganaha, Y., Tokokawa, N., Miyazato, T., Miyazato, M., Kohatsu, T., Shimabukuro, H., Tamashiro, J., 1996. Optical Absorption of Single Crystals of Y3A15O12 and Y3Al5O12:Nd3+ in the UV Fundamental Absorption Edge Region. J. Phys. Soc. Jpn. 65, 1106-1113.

[20] Pustovarov, V.A., Ivanov, V.Yu.,Kirm, M., Korotaev, A.V., Kruzhalov, A.V., Zimmerer, G., Zinin, E.I., 2001. Time-resolved luminescent VUV spectroscopy of F- and F+ -centers in single $\mathrm{BeO}$ crystals, Nucl. Instr. Meth. A 470, 353-357. 